\title{
Meilensteine der Gesundheitspolitik und seltene Erkrankungen
}

\author{
Milestones of Health Policy and Rare Diseases
}

\section{T. Klingebiel ${ }^{1}$ \\ U. Creutzig ${ }^{2}$ \\ A. Christaras ${ }^{3}$ \\ U. Nowak-Göttl ${ }^{4}$ \\ U. Göbel ${ }^{5}$}

Institutionen

${ }^{1}$ Vorsitzender der Gesellschaft für Pädiatrische Onkologie und Hämatologie

${ }^{2}$ Geschäftführerin der Gesellschaft für Pädiatrische Onkologie und Hämatologie

${ }^{3}$ HELIOS Klinikum Krefeld

${ }^{4}$ Universitätskinderklinik und Poliklinik Münster, Hämatologie/Onkologie

${ }^{5}$ ESPED-Geschäftsstelle, Koordinierungszentrum für Klinische Studien, HeinrichHeine-Universität Düsseldorf

Bibliografie

DOI http://dx.doi.org/

$10.1055 / \mathrm{s}-0030-1252048$

Klin Padiatr 2010; 222: 121-123

(c) Georg Thieme Verlag KG

Stuttgart · New York

ISSN 0300-8630

Korrespondenzadresse

Prof. Dr. Ulrich Göbel

Universitätsklinikum

Heinrich-Heine-Universität

Düsseldorf

ESPED-Geschäftsstelle

Koordinierungszentrum für

Klinische Studien

Moorenstraße 5

40225 Düsseldorf

Tel.: + 49/211/8116263

Fax: $+49 / 211 / 8116262$

heinrich@med.uni-

duesseldorf.de
Die Behandlung von Patienten unterliegt entsprechend dem wissenschaftlichen Fortschritt einem stetigen Wandel, von dem Kinder mit onkologischen und hämatologischen Erkrankungen in ganz besonderer Weise durch die kooperativen, interdisziplinären und multizentrischen Therapieoptimierungsstudien profitiert haben [5]. Dies setzt auch bei den kooperierenden Fachdisziplinen die Bereitstellung der erforderlichen Ressourcen voraus [3]. Es überleben nicht nur zunehmend mehr Patienten durch eine an das individuelle Rückfallrisiko adaptierte Therapie, sondern die Modalitäten für ein krankheitsfreies Langzeitüberleben und die Normalisierung der gesundheitsbezogenen Lebensqualität sind Inhalte weiterführender klinischer Forschung $[7,25]$. Über Jahrzehnte haben sich hierbei Strukturen für die klinische Forschung etabliert, die wesentlich durch begutachtete Drittmittel oder Spenden finanziert sind $[6,16,22,27]$. Im zurückliegenden Jahrzehnt sind die Rahmenbedingungen durch die Gesetzgebung direkt oder indirekt durch die Schaffung neuer Strukturen gravierend geändert worden, wie vier Hinweise belegen:

- Die anhaltend defizitäre Finanzlage der gesetzlichen Krankenkassen hat im Jahr 2002 zur Einführung eines neuen Verfahrens zur Finanzierung der stationären Behandlungen und der Krankenhäuser veranlasst $[1,11]$. Nach dem Vorbild anderer Länder wird durch einheitliche Fallpauschalen eine leistungsbezogene Vergütung ohne Wettbewerbsverzerrungen in einem mehrjährigen Angleichungsprozess angestrebt [23]. Da dieses G-DRG-System als selbstlernend konzipiert ist, unterliegt es einem immerwährenden Anpassungsprozess.

- Mit der 12. Novelle des Arzneimittelgesetzes ist 2004 die EU-Richtlinie zur „Good Clinical Practice“ umgesetzt worden, die für Therapieoptimierungsstudien die gleichen systematischen Wirksamkeits- und Unbedenklichkeitsprüfungen wie für neu zuzulassende Medikamente vorschreibt $[31,33]$.

- Um in Deutschland flächendeckend eine hochwertige Versorgung für kinderonkologische Erkrankungen sicherzustellen, sind 2006 durch die Gremien des Gemeinsamen Bundesausschusses Maßnahmen zur Qualitätssicherung beschlossen und entsprechend der vorgegebenen Meilensteine kontinuierlich umgesetzt worden [30]*. Dies hat zu einer weiteren Zentralisierung der kinderonkologischen Behandlung geführt, ohne dass entsprechend der zusätzlichen Leistungen das Budget des jeweiligen Klinikums aufgestockt worden ware.

- Das neugegründete Institut für Qualität und Wirtschaftlichkeit im Gesundheitswesen (IQWiG) [17] hat im Jahr 2009 die kinderonkologische Behandlung in Deutschland umfassend evaluiert und die Versorgungsqualität in Bezug auf das Überleben als sehr gut bezeichnet; Indikatoren für die Struktur- und Prozessqualität würden jedoch dringend benötigt [18]. Der Verzicht auf die Finanzierung von Leistungen über Drittmittel, die der direkten Versorgung krebskranker Kinder dienen, ist Gegenstand aktueller Verhandlungen mit dem Bundesministerium für Gesundheit.

Desungeachtet enthält die Vorausschau auf das neue Jahrzehnt erhebliche Imponderabilien:

Die Zahl patentgeschützter Neuentwicklungen zur zielgerichteten Therapie von Krebserkrankungen nimmt in den letzten Jahren deutlich zu, wodurch mit zusätzlichen Kosten für das Gesundheitssystem zu rechnen ist. Diese neuen und neuartigen Präparate bedürfen der sorgfältigen Prüfung hinsichtlich ihrer Wirksamkeit, Verträglichkeit und Effizienz. Bei krebskranken Kindern und Jugendlichen ergeben sich in Anbetracht der erfreulich hohen Heilungsraten einerseits und der niedrigen Fallzahlen andererseits sowie der gesetzlichen Rahmenbedingungen schwere und unüberwindlich erscheinende Hindernisse $[5,7,10,19,24,33]$. Gleiches gilt für die translationale Forschung mit der Herstellung neuartiger Therapeutika, die in der Onkologie oder Hämatologie durch die Modifikation patienteneigenen Materials zunehmend die hochgesteckten Prämissen einer individualisierten Therapie erfüllt [12].

Die Verleihung des Leibniz-Preises der Deutschen Forschungsgemeinschaft am 15.3.2010 an Herrn Professor Christoph Klein, Hannover, u.a. zur Behandlung des Wiskott-Aldrich-Syndroms mittels gentherapeutisch modifizierter autologer Blutstammzellen ist das weit herausragende Beispiel für die gelungene Überwindung aller Hindernisse für ein innovatives Behandlungskonzept bei einer sehr seltenen Erkrankung [4], wozu die Gesellschaft für Pädiatrische Onkologie und Hämatologie dem Kind-Philipp-Preisträger von 2006 herzlich gratuliert [21]; damals sind seine For-

\footnotetext{
*Vereinbarung des Gemeinsamen Bundesausschusses über Maßnahmen zur Qualitätssicherung für die stationäre Versorgung von Kindern und Jugendlichen mit hämato-onkologischen Krankheiten gemäß § 137 Abs. 1, Satz 3 Nr. 2 SGB V für nach $§ 108$ SGB V zugelassenen Krankenhäuser (Vereinbarung zur Kinderonkologie) vom 16. Mai 2006, In-Kraft-Treten am 1. Januar 2007.
} 
schungen zur schweren angeborenen Neutropenie (Kostmann disease), einer gleichfalls sehr seltenen Erkrankung, ausgezeichnet worden [20].

Die erfolgreiche Behandlung des Wiskott-Aldrich-Syndrom als monogenetischer Erkrankung stellt ebenso wie eine unheilbare Krebserkrankung, bei der alle erfolgversprechenden Therapiemaßnahmen versagt haben, eine Schwarz-Weiß-Situation dar, da es leicht zu überprüfende Wirksamkeitskriterien gibt und die Lebenserwartung ohne kurative Therapie überschaubar ist; insofern lässt sich die Kausalität der durchgeführten neuen Therapie für die verlängerte Lebenserwartung ohne den geringsten Restzweifel eindeutig belegen. Sehr viel schwieriger ist die Beweisführung bei seltenen Erkrankungen, die multifaktoriell bedingt sind und nicht zu einer stark verkürzten Lebenserwartung, aber möglicherweise lebenslänglichen Beeinträchtigung führen. Hier stimmen 3 Entwicklungen hoffnungsvoll:

- Anlässlich des Tages für die seltene Erkrankung ist am 28.2.2010 der Eva-Luise-Köhler-Forschungspreis an Frau Dr. Karin Jurkat-Rott und Herrn Dr. Marc-André Weber für ihren innovativen Therapieansatz zur Behandlung von Patienten mit hypokaliämischer periodischer Paralyse verliehen worden [28].

- Das Bundesgesundheitsministerium hat kürzlich ein Aktionsbündnis für Menschen mit seltenen Erkrankungen (NAMSE bzw. ACHSE) vorgestellt, das ein gemeinsames, koordiniertes und zielorientiertes Handeln der maßgeblichen Akteure im deutschen Gesundheitswesen ermöglichen soll [26, 28,32].

- Die Europäische Kommission hat ihren Mitgliedstaaten empfohlen, bis spätestens 2013 nationale Aktionspläne für Menschen mit seltenen Erkrankungen zu erstellen [8].

Zur Realisierung der Pläne bedarf es der langfristigen Bereitstellung entsprechender Ressourcen, die unter Berücksichtigung von einheitlichen, überprüfbaren und regelmäßig zu kontrollierenden Qualitätskriterien einzuwerben sind. Hierbei ist zuvorderst der verfügbare Sachverstand einzubringen, wie er beispielhaft in der Arbeitsgemeinschaft der Wissenschaftlichen Medizinischen Fachgesellschaften gebündelt ist [2]. Weiterhin sind biometrische Verfahren zu propagieren, die als Alternativen zu randomisierten Prüfungen, die aufgrund zu geringer Patientenzahlen nicht durchführbar sind, akzeptiert werden [10,24]. Zu denken ist hier an die Definition von Patienten mit besonders guter und besonders schlechter Prognose, um selektiv an kleinen Patientengruppen die Vorzüge neuer Therapiemaßnahmen rational und rationell zu überprüfen. Dies setzt die Definition von allgemein anerkannten Risikofaktoren und prospektiv angelegte klinische Register voraus, deren Nutzen belegt ist, aber deren langfristige Finanzierung einer Lösung bedarf [15]. Um die pathogenetischen Ursachen untersuchen und die biometrisch gesicherte Wirksamkeit molekularbiologisch nachvollziehen zu können, sind entsprechende Biomaterialbanken anzulegen, die erst durch die Kooperation mit entsprechenden diagnosebezogenen klinischen Registern ihren besonderen Wert erfahren [9]. Darüber hinaus gilt es, die Kooperation mit nahe verwandten Fachdisziplinen wie der pädiatrischen Immunologie [14] und der pädiatrischen Hämostaseolgie [13] zu vertiefen, wofür der diesjährige Leibniz-Preis für Medizin das herausragende Beispiel ist.

Wie groß der Bedarf für einheitliche Dokumentationen ist, wird beispielhaft an der Metaanalyse zur Thrombose der Aorta bei Neugeborenen (s. S. 134-139) sichtbar. Die Recherche der englischsprachigen Literatur über 20 Jahre erbrachte insgesamt nur 38 informative Veröffentlichungen, die insgesamt nur zu $148 \mathrm{~Pa}$ - tienten berichten; bei 68 Patienten fehlt die Angabe des Geschlechts bzw. bei 32 Patienten die Angabe des Geburtsgewichts. Auch wenn die Bildgebung zum Nachweis der Thrombose in jedem Einzelfall dokumentiert ist, bleiben bei dieser Datenlage gewisse Zweifel hinsichtlich der Vollständigkeit möglicher oder sicherer Risikofaktoren bzw. der Häufigkeit und Schwere von Spätfolgen. Trotzdem haben die Autoren in verdienstvoller Weise einen diagnostisch-therapeutischen Algorithmus erstellt, da unbehandelt schwere Thrombosen der Aorta zu einer erheblichen Morbidität führen können und die thrombolytische Therapie selbst aber als potenziell riskant einzustufen ist.

Trotz der kleinen Fallzahl gelingt es somit, Empfehlungen auch für seltene gutartige Erkrankungen zu erarbeiten, die wie zum Beispiel bei seltenen Krebserkrankungen des Kindes- und Jugendalters zu einer starken Zunahme der Überlebensraten geführt haben $[5,7,29]$. Dies ist wesentlich erleichtert, wenn Kooperationen mit optimal funktionierenden epidemiologischen Registern möglich sind [7,19].

In Anbetracht der knappen Ressourcen im Gesundheitswesen sind rational begründete Therapien in möglichst rationeller Weise auch bei seltenen Erkrankungen zu prüfen, wozu das vorliegende Heft in Sinne der Bestandsaufnahme unterschiedlicher Erkrankungen mit onkologischem, hämatologischem oder hämastaseologischem Hintergrund einen Beitrag liefern will.

\section{Literatur}

1 Artikel 1. GKV-Modernisierungsgesetz vom 14 November 2003 (BGBl. I S. 2190)

2 AWMF. Erarbeitung von Leitlinien für Diagnostik und Therapie: Methodische Empfehlungen AWMF Online http://www.uniduesseldorf. de/AWMF/li/ll_metho.htm 2004

3 Bölling T, Ernst I, Könemann S et al. Pediatric Radiation Oncology in Germany: A study of availability and application. Klin Padiatr 2008; 220: $178-182$

4 Boztug K, Dewey RA, Klein C. Development of hematopoietic stem cell gene therapy for Wiskott-Aldrich syndrome. Curr Opin Mol Ther. 2006; 8: 390-395

5 Creutzig $U$, Henze G, Bielack $S$ et al. Krebserkrankungen im Kindesalter. Erfolg durch einheitliche Therapiekonzepte seit 25 Jahren. Deutsches Ärzteblatt 2003; 100: A842-A852

6 Creutzig $U$, Henze G, Herold $R$ et al. Quality management for clinical trials within the German Competence Network Paediatric Oncology and Haematology. Onkologie 2005; 28: 333-336

7 Debling D, Spix C, Blettner M et al. Cohort of Long-term Survivors after Childhood Cancer at the German Childhood Cancer Registry. Klin Padiatr 2008; 220: 372-378

8 Empfehlung des Rates vom 8. Juni 2009 für eine Maßnahme im Bereich seltener Krankheiten (2009/C 151/02) . Amtsblatt der Europäischen Union DE 3.7.2009 C 151/7

9 Ernestus K, Pietsch T, Gessler M et al. Biomaterialbanken für embryonale Tumoren: Struktur, Nutzen und Risiken. Klin Padiatr 2006; 218: 132-138

10 European Medicines Agency (EMEA). ICH Topic E 9. Statistical principles for clinical trials EMEA http://www.emea.europa.eu/pdfs/human/ich/ 036396en.pdf 2006

11 Gesetz zur Einführung des Diagnose-orientierten Fallpauschalensystems für Krankenhäuser (Fallpauschalengesetz - FPG) BGBl I Nr. 27, S. 1412-1437. 29. April 2002

12 Göbel U. Bitte einen Gruppenfahrschein für „From Bed to Bench“ und wieder zurück !. Klin Padiatr 2005; 217: 307-309

13 Göbel U, Kontny U (Hrsg.). Forschungsergebnisse der pädiatrischen Onkologie, Hämatologie und Hämostaseologie 40. Klin Padiatr 2009; 221: 131-216

14 Göbel, Lehrnbecher T, Niehues T (Hrsg.). Forschungsergebnisse der pädiatrischen Onkologie, Hämatologie und Immunologie 38. Klin Padiatr 2008; 220: 327-402

15 Göbel U, Witt O. Das Dilemma der klinischen Register in der pädiatrischen Onkologie und Hämatologie. Klin Padiatr 2008; 220: 129-133

16 Herold $R$, Reiche $R$, Creutzig $U$ et al. Stand und Bewertung der Strukturen und Ausstattung pädiatrisch-onkologisch-hämatologischer Kliniken. Klin Padiatr 2007; 219: 380-390 
17 Institut für Qualität und Wirtschaftlichkeit im Gesundheitswesen (IQWiG). Allgemeine Methoden. Version 3.0 vom 25.05.2008. 2008 http://www.iqwig.de/download/IQWiG_Allgemeine_Methoden_ Entwurf_V-3-0.pdf

18 Institut für Qualität und Wirtschaftlichkeit im Gesundheitswesen (IQWiG), Hrsg. Qualität der pädiatrisch-hämatologisch-onkologischen Versorgung Abschlussbericht V06-01, Version 1.0 vom 13.08.2009. IQWiG-Berichte - Jahr: 2009 Nr. 62

19 Kaatsch P, Steliarova-Foucher E, Crocetti E et al. Time trends of cancer incidence in European children (1978-1997): report from the ACCIS project. Eur J Cancer 2006; 42: 1961-1971

20 Klein C, Grudzien M, Appaswamy G et al. HAX1 deficiency causes autosomal recessive severe congenital neutropenia (Kostmann disease). Nat Genet 2007; 39: 86-92

21 Klingebiel T, Creutzig U, Henze G et al. Kind-Philipp-Symposium zu Ehren des Stifters Herrn Dr-Ing Walter Reiners und Verleihung des Kind-Philipp-Preises 2008. Klin Padiatr 2009; 221: 327-331

$22 \mathrm{KPOH}$. Kompetenznetz Pädiatrische Onkologie und Hämatologie (KРOH) http://www.kinderkrebsinfo.de/e216 2008

23 Lüngen $M$, Lauterbach KW. Applicability of diagnostic related groups (DRG) in an international comparison. Chirurg 2000; 71: 1288-1295

24 Ohmann C. Klinische Studien in der Pädiatrie: Herausforderungen und aktuelle Entwicklungen. Klin Padiatr 2008; 220: 221-223

25 Peeters $J$, Meitert J, Paulides $M$ et al. Health-related quality of life (HRQL) in ALL-patients treated with chemotherapy only - A report from the Late Effects Surveillance System in Germany. Klin Padiatr 2009; 221: 156-161
26 Pressemitteilung: Nationales Aktionsbündnis für Menschen mit Seltenen Erkrankungen (NAMSE). http://www.bmg.bund.de/SharedDocs/ Downloads/DE/Presse/Presse-2010/pm-08-03-10-NAMSE

27 Reiche $R$, Herold $R$. Strukturen in der pädiatrischen Onkologie und Hämatologie, Ergebnisse der wissenschaftlichen Begleitung der Jahre 2000-2006. Bonn: Js. Karthaus GmbH \& Co; 2007

28 Schmitt-Sausen $N$. Mehr Aufmerksamkeit für Waisenkinder der Medizin. Deutsches Ärzteblatt 2010; 107: B 381

29 Thiele KP, Rheineberger $P$. „Unmöglich“ gibt es nicht. Deutsches Ärzteblatt 2003; 100: A1044-A1046

30 Verfahrensordnung des Gemeinsamen Bundesausschusses, zuletzt geändert am 18. April 2006, veröffentlicht im Bundesanzeiger 2006, S. 4876, in Kraft getreten am 7. Juli 2006. G-BA 2006

31 Verordnung über die Anwendung der Guten Klinischen Praxis bei der Durchführung von klinischen Prüfungen mit Arzneimitteln zur Anwendung am Menschen (GCP-V). DCP-Verordnung vom 9. August 2004 (BGBL. I S. 2081), zuletzt geändert durch Artikel 4 der Verordnung vom 3. November 2006 (BGBL. I S. 2523). Bundesministerium der Justiz 2006

32 www.achse-online.de. Zugriff am 21.03.2010 10:48 CET

33 Zwölftes Gesetz zur Änderung des Arzneimittelgesetzes vom 30. Juli 2004. BGBl I 05. August 2004. Nr. 41, 2031-2053 